\title{
Impacto clínico y epidemiológico de las infecciones por varicela en niños previo a la introducción de la vacuna al Calendario Nacional de Argentina
} Clinical and epidemiological impact of varicella infection in children prior to the introduction of the varicella vaccine in the national immunization schedule of Argentina

\author{
Dra. Silvina E. Neyro ${ }^{a}$, Dr. Fausto M. Ferolla ${ }^{a, b}$, Dra. Claudia Molise, \\ Dra. Patricia Stach ${ }^{b}$, Dr. Pablo Romanoc, Dra. Silvia Maroned, Dr. Arturo de Mena ${ }^{a}$, \\ Dra. Fabiola Plat ${ }^{a}$, Dra. Carla Voto ${ }^{a}$, Dr. Pedro Soto ${ }^{a}$, Dra. Laura Bustos ${ }^{c}$, \\ Dra. Cecilia Clavijoc ${ }^{c}$ Dr. Daniel Murgo ${ }^{d}$, Dr. Daniel Dozoretz ${ }^{d}$, Dr. Jorge Fiorentino ${ }^{b} y$ \\ Dr. Eduardo L. Lópeza
}

\section{RESUMEN}

Introducción. En Argentina, se estiman 400000 casos anuales de varicela. Dado el subregistro de casos existentes, la carga de enfermedad real se desconoce.

Objetivo. Evaluar la carga de enfermedad por varicela antes de la introducción de la vacuna al Calendario Nacional.

Materiales y métodos. Estudio retrospectivo, analítico, observacional, realizado en tres centros asistenciales del país. Revisión de los registros de consultas ambulatorias a los Servicios de Urgencias y de las historias clínicas de las internaciones por varicela en pacientes $<18$ años. Período: 1/2011-12/ 2013.

Resultados. Fueron asistidas un total de 382782 consultas ambulatorias; $3367(0,88 \%)$ correspondieron a consultas por varicela; el $57,6 \%, \leq 4$ años. Requirieron internación 164 $(4,9 \%)$ con una tasa de hospitalización global de 65,3/10 000 hospitalizados/año (IC $95 \%$ : $55,4-76,5)$; tasa de hospitalización en niños sanos: 57,2/ 10000 (IC 95\%: 67,7-48,0); mediana de edad: 31,5 meses. Las causas de internación más frecuentes fueron infecciones de piel y/o partes blandas $(61,1 \%)$ y respiratorias $(10,1 \%)$. El 54,3\% recibió tratamiento con aciclovir, y el $73,1 \%$, con antibióticos. Presentaron bacteriemia $5 / 67(7,5 \%)$, todas por cocos $\operatorname{Gram}(+)$ y en inmunocompetentes. De los 19 pacientes inmunocomprometidos, el 36,8 \% tuvo complicaciones (5 infecciones de piel y/o partes blandas y 2 neumonías). La mediana de días de internación fue 4 , significativamente más prolongadaen pacientesinmunocomprometidos. Un paciente requirió cuidados intensivos. No hubo fallecidos.

Conclusión. La carga de enfermedad registrada fue significativa, con impacto considerable en pacientes sin patología de base.

Palabras clave: varicela, pediatría, carga de la enfermedad, atención ambulatoria, hospitalización.

http: / / dx.doi.org/10.5546/ aap.2019.12

Texto completo en inglés:

http:/ / dx.doi.org/10.5546/aap.2019.eng.12
Cómo citar: Neyro SE, Ferolla FM, Molise C, Stach $\mathrm{P}$, et al. Impacto clínico y epidemiológico de las infecciones por varicela en niños previo a la introducción de la vacuna al Calendario Nacional de Argentina. Arch Argent Pediatr 2019;117(1):12-18.

\section{INTRODUCCIÓN}

La varicela es una enfermedad infectocontagiosa aguda que resulta de la infección primaria por el virus varicela-zóster. Es una de las enfermedades exantemáticas más frecuentes de la niñez. La mayoría de los casos ocurren durante la primera década de la vida y solo el $10 \%$ de los adultos jóvenes persisten susceptibles. ${ }^{1}$ Es considerada una enfermedad benigna de la infancia, pero puede producir complicaciones, principalmente, en los adultos ${ }^{2-5} \mathrm{y}$ en los inmunocomprometidos. ${ }^{2,4,6}$ Los niños sanos también están expuestos a desarrollar complicaciones (desde infecciones de piel y partes blandas -IPPB- a infecciones sistémicas con alta morbimortalidad).,2,4,-8

En Argentina, el Sistema Nacional de Vigilancia en Salud registra 150 000-200 000 casos / año de varicela, con una tasa aproximada de 250 450 casos / 100000 habitantes. Sin embargo, se estima que ocurren más de 400000 casos nuevos cada año. ${ }^{9}$ Este subregistro de casos genera desconocimiento sobre el real impacto que conlleva la carga de enfermedad por varicela en Argentina, en cuanto a 
consultas ambulatorias, tasas de hospitalización (TH) y complicaciones. Esta situación se ha evidenciado también en otros países de la región. ${ }^{2}$

Ante esta escasez de datos, se considera relevante obtener evidencia para conocer la magnitud del problema en un período previo a la introducción de la vacuna al Calendario Nacional y constituir la línea de base para evaluar en forma posterior el impacto que la estrategia nacional de vacunación genere. En Argentina, la vacuna contra la varicela fue incluida en el Calendario Nacional de Vacunación en julio de 2015, en esquema de dosis única (15 meses de vida). ${ }^{9}$

El objetivo de este estudio fue evaluar la carga de enfermedad por varicela en tres centros asistenciales de Argentina antes de la introducción de la vacuna.

\section{MATERIALES Y MÉTODOS}

Estudio retrospectivo, analítico y observacional desarrollado en 3 hospitales de Argentina: Hospital de Niños "Dr. Ricardo Gutiérrez" (hospital pediátrico de $3^{\text {er }}$ nivel, público, de la Ciudad Autónoma de Buenos Aires), Hospital Interzonal General de Agudos "Evita" (hospital público, de Lanús, provincia de Buenos Aires) y Hospital "Marcial V. Quiroga" (hospital general, público, de la provincia de San Juan).

Los datos fueron recolectados a través de la revisión de libros de registro de consultas ambulatorias realizadas en los Servicios de Urgencias, así como de historias clínicas de pacientes menores de 18 años asistidos o internados por varicela o sus complicaciones, durante el período comprendido entre enero de 2011 y diciembre de 2013.

El diagnóstico de varicela fue realizado por pediatras de los Servicios de Urgencias o de salas de internación de Clínica Pediátrica, sobre la base de los hallazgos clínicos y evolutivos de la enfermedad. Se recabó información sobre características demográficas, presencia/tipo de comorbilidad, antecedente de vacunación, presencia/tipo de complicación, días de hospitalización, resultados de análisis de laboratorio y de estudios por imágenes (en caso de haber sido solicitados como práctica de rutina), necesidad de procedimientos quirúrgicos, uso de antibióticos y antivirales.

El presente estudio fue aprobado por los Comités de Ética de los centros participantes.

\section{Análisis estadístico}

Para el análisis estadístico, se utilizó el programa Stata versión 11.2. Los resultados se expresaron como media aritmética o mediana y desviación estándar en el caso de las variables cuantitativas y como porcentaje en el caso de las variables cualitativas. Las tasas fueron calculadas con sus respectivos intervalos de confianza (IC $95 \%$ ). Se utilizaron diferentes pruebas de asociación estadística de acuerdo con el tipo de variables: para la comparación de proporciones, se utilizó el test de chi cuadrado o el test de Fisher, y la $t$ de Student o la U de Mann-Whitney para la comparación de medias, según correspondiera. Se consideró estadísticamente significativo un valor de $p$ menor de 0,05 .

\section{RESULTADOS \\ Consultas ambulatorias por varicela}

Durante el período de estudio, se realizaron 382782 consultas a los Servicios de Urgencias; en 3367 (0,88\%), se diagnosticó varicela, lo que representó una incidencia de 88 casos / 10000 consultas ambulatorias / año (IC $95 \%$ : 85-91) (Tabla 1). El 57,6 \% fueron menores de 4 años, con una mediana de 48 meses (rango intercuartilo [RIC]: 24-72); el 50,1\%, de sexo femenino. Analizando los subgrupos de edad, en menores de 1 año, se diagnosticaron 333 casos (28 se internaron); en el grupo de 1-4 años, 1607 casos (79 se internaron); de 5-14 años, 1401 casos (43 se internaron) y, de 15-18 años, 26 casos (3 se internaron).

Se evidenció una estacionalidad marcada de las consultas por varicela, que predominaron entre los meses de junio y noviembre, correspondientes al invierno y parte de la primavera (Figura 1).

La aparición de exantema fue el motivo más frecuente de consulta $(n=3178 ; 94,4 \%)$. Otros fueron fiebre, dificultad respiratoria, sobreinfección bacteriana de las lesiones, control evolutivo.

Eran previamente sanos $3339(99,2 \%)$ niños. Solo $28(0,8 \%)$ padecían alguna comorbilidad, y las más frecuentes eran las oncológicas $(n=6$; $21,4 \%)$ y las reumatológicas $(n=6 ; 21,4 \%)$, seguidas por otras inmunodeficiencias $(n=5$; $17,8 \%)$ y enfermedades hematológicas ( $\mathrm{n}=$ 4; 14,2 \%); las restantes: 2 epilepsias, 1 asma, 1 dermatitis atópica y 1 cardiopatía, 1 nefropatía y 1 hepatopatía crónicas.

Al momento de la consulta ambulatoria, 401 $(11,9 \%)$ pacientes presentaban complicaciones asociadas a la varicela, 33 de ellos más de una. Las más frecuentes fueron las IPPB $(\mathrm{n}=321 ; 80 \%)$, seguidas por las respiratorias $(\mathrm{n}=30 ; 7,5 \%)$ y neurológicas $(n=13 ; 3,2 \%)$. 


\section{Internaciones por varicela}

De los 3367 pacientes con varicela que consultaron a los Servicios de Urgencias, 164 se internaron (4,9\%; IC $95 \%$ : 4,2-5,6): el 8,4 \% de los menores de 1 año (IC $95 \%$ : 5,7-11,9), el $4,9 \%$ (IC $95 \%: 3,9-6,1$ ) de los niños de 1-4 años, el 3,1 \% (IC 95\%: 2,2-4,1) de los de 5-14 años y el 11,5\% (IC $95 \%$ : 2,4-30,2) de los mayores de 14 . Se analizaron 153 pacientes (el 93,3\% del total), dado que 11 se derivaron a otros centros. La TH global por varicela fue de 65,3 casos $/ 10000$ internaciones / año (IC $95 \%$ : 55,4-76,5) (Tabla 1).
Ochenta $(52,6 \%)$ pacientes internados eran varones, con una mediana de edad de 31 meses (RIC: 15-64), significativamente más alta en aquellos con inmunocompromiso (mediana de 67 meses, RIC: $33-120, p<0,001$ ). Solo $3 / 102$ $(2,9 \%)$, previamente sanos, presentaban el antecedente de estar vacunados, con una única dosis.

La fuente de contagio era conocida en $50(32,9 \%)$ pacientes: en $38(76 \%)$, fue un caso primario intrafamiliar; únicamente $3(6 \%)$ habían recibido algún tipo de profilaxis posexposición.

TABLA 1. Consultas ambulatorias e internaciones por varicela $y$ totales

\begin{tabular}{|c|c|c|c|c|c|c|c|c|c|c|c|c|c|}
\hline & \multicolumn{4}{|c|}{$\begin{array}{c}\text { Hospital de Niños } \\
\text { Dr. Ricardo Gutiérrez }\end{array}$} & \multicolumn{4}{|c|}{$\begin{array}{l}\text { Hospital General } \\
\text { de Agudos "Evita" }\end{array}$} & \multicolumn{4}{|c|}{$\begin{array}{c}\text { Hospital } \\
\text { Marcial Quiroga }\end{array}$} & \multirow{2}{*}{$\begin{array}{c}\text { TOTAL } \\
2011-2013\end{array}$} \\
\hline & 2011 & 2012 & 2013 & Total & 2011 & 2012 & 2013 & Total & 2011 & 2012 & 2013 & Total & \\
\hline $\begin{array}{l}\text { Consultas } \\
\text { ambulatorias } \\
\text { por varicela }\end{array}$ & 602 & 422 & 811 & 1835 & 179 & 171 & 243 & 593 & 480 & 184 & 275 & 939 & 3367 \\
\hline $\begin{array}{l}\text { Consultas } \\
\text { ambulatorias } \\
\text { totales }\end{array}$ & 58643 & 65972 & 60646 & 185261 & 25716 & 26126 & 27512 & 79354 & 41589 & 39747 & 36831 & 118167 & 382782 \\
\hline $\begin{array}{l}\text { Consultas } \\
\text { por varicela/ } \\
10000 \text { consultas } \\
\text { totales }\end{array}$ & 103 & 64 & 134 & 99 & 70 & 65 & 88 & 75 & 115 & 46 & 75 & 79 & 88 \\
\hline $\begin{array}{l}\text { Internaciones } \\
\text { por varicela }\end{array}$ & 32 & 27 & 38 & 97 & 5 & 6 & 12 & 23 & 19 & 9 & 16 & 44 & 164 \\
\hline $\begin{array}{l}\text { Internaciones } \\
\text { totales }\end{array}$ & 5310 & 5778 & 5603 & 16691 & 1492 & 1389 & 1423 & 4304 & 1265 & 1450 & 1390 & 4105 & 25100 \\
\hline $\begin{array}{l}\text { Internaciones } \\
\text { por varicela/ } \\
10000 \text { int. totales }\end{array}$ & 60 & 47 & 68 & 58 & 34 & 43 & 84 & 53 & 150 & 62 & 115 & 107 & 65,3 \\
\hline
\end{tabular}

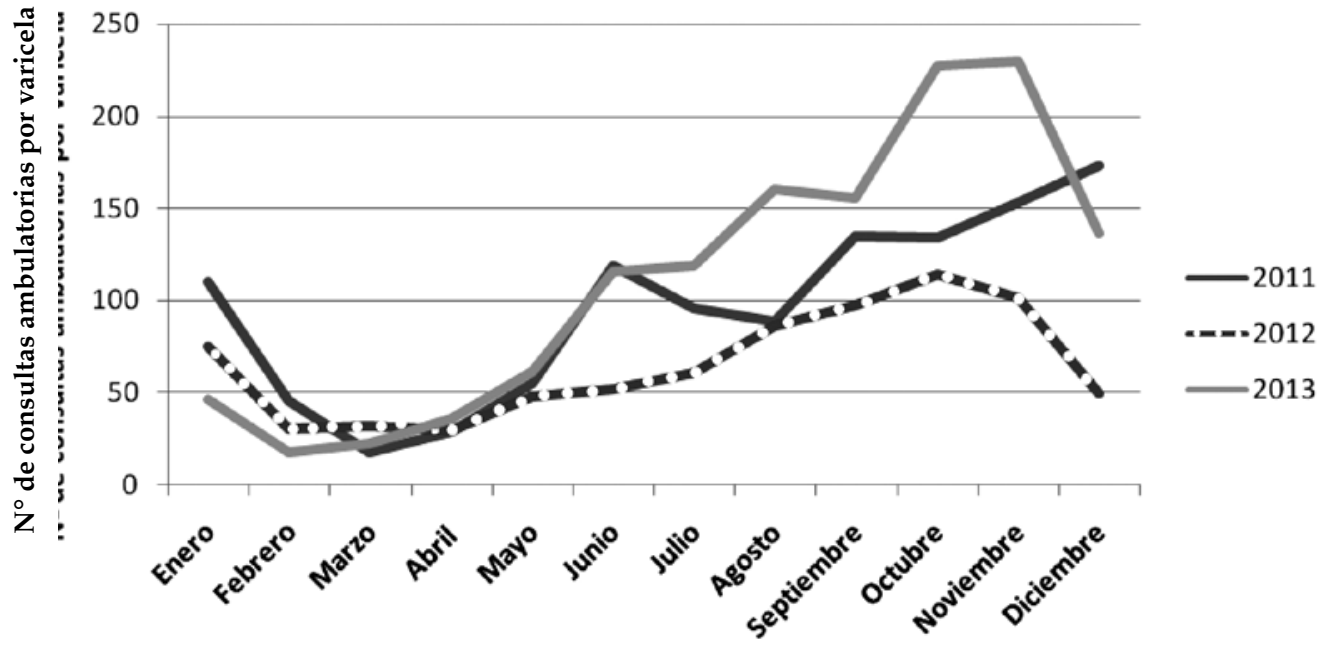


La mediana de tiempo de evolución del exantema al momento del ingreso hospitalario fue de 4 días (RIC: 2-5 días) y de la duración de la fiebre fue de 2 días (RIC: 1-3 días).

Del total de internados, $134(87,6 \%)$ eran inmunocompetentes y fueron hospitalizados por complicaciones asociadas a varicela (Tabla 2 ). Considerando solo a los niños sanos, la tasa de internación fue de 57,2/10 000 hospitalizaciones / año (IC $95 \%$ : 67,7-48,0). Diecinueve pacientes $(12,4 \%)$ eran inmunocomprometidos (en 10, corticoterapia prolongada; en 5, enfermedad hematooncológica; en 1, trasplante hepático; en 3, otros tratamientos inmunosupresores).

A diferencia de lo ocurrido en niños sanos, del total de inmunocomprometidos internados, solo 7 $(36,8 \%)$ presentaron complicaciones infecciosas: 5, IPPB, y 2, neumonía. En la población estudiada, no hubo diferencias significativas con respecto a la edad y al tiempo de evolución de la enfermedad, en relación con el tipo de complicación que presentaron.

Se les realizó un hemograma al ingresar a $138(90,2 \%)$ niños, con un recuento de leucocitos de $10567 \pm 5750 / \mathrm{mm}^{3}$, y un hepatograma a 60 pacientes. En $9(15 \%)$, se constató un aumento transitorio de las enzimas hepáticas mayor de 80 U / 1 . Se les solicitó una radiografía de tórax a $60(39,2 \%)$ pacientes, y resultaron patológicas 18 (30\%): 10 con neumonitis, 8 con neumonía. En 4 de 5 ecografías de partes blandas realizadas, se confirmó el diagnóstico de celulitis. A 2 casos con encefalitis se les solicitó una tomografía computada, sin imágenes patológicas. A 9 pacientes con síntomas neurológicos, se les

TABla 2. Complicaciones de varicela que motivaron la internación en pacientes inmunocompetentes

\begin{tabular}{lc}
\hline Complicación & $\mathbf{n}=\mathbf{1 3 4}(\mathbf{\%})$ \\
\hline Infección de piel y partes blandas & $\mathbf{9 3 ( 6 0 , 8 )}$ \\
Impétigo & $66(43,1)$ \\
Celulitis & $15(9,8)$ \\
Eritrodermia & $8(5,2)$ \\
Celulitis orbitaria/preseptal & $4(2,6)$ \\
Compromiso respiratorio & $\mathbf{1 8 ( 1 1 , 8 )}$ \\
Neumonitis & $10(6,5)$ \\
Neumonía & $8(5,2)$ \\
Compromiso neurológico & $\mathbf{1 3}(8,5)$ \\
Ataxia & $5(3,3)$ \\
Convulsión febril & $4(2,6)$ \\
Encefalitis & $4(2,6)$ \\
Varicela hemorrágica/púrpura & $\mathbf{5 ( 3 , 3 )}$ \\
Sepsis & $\mathbf{3 ( 2 , 0 )}$ \\
Vómitos/diarrea & $\mathbf{2 ( 1 , 3 )}$ \\
\hline
\end{tabular}

realizó una punción lumbar: en uno de ellos, el líquido cefalorraquídeo presentó celularidad aumentada (52 leucocitos $/ \mathrm{mm}^{3}$ ) y, en otro, hiperproteinorraquia $(94 \mathrm{mg} / \mathrm{dl})$. Ninguno de los casos tuvo diagnóstico microbiológico en el líquido cefalorraquídeo.

En $18(94,7 \%)$ inmunocomprometidos y en $51(38,1 \%)$ inmunocompetentes, se indicó el tratamiento con aciclovir, durante una mediana de 7 días (RIC: 6-10 y 5-7, respectivamente).

Se medicaron con antitérmicos $93(60,8 \%)$ pacientes, durante una mediana de 2 días (RIC: 1-3); en 21 (22,6\%) de estos, se indicó ibuprofeno y no se observaron eventos adversos asociados a su uso.

Se realizaron cultivos bacteriológicos en 70 pacientes: 67 pares de hemocultivos, $5(7,5 \%)$ de ellos positivos (2 Streptococcus pyogenes, 2 Staphylococcus aureus meticilino resistente -SAMR-, 1 Staphylococcus aureus meticilino sensible -SAMS-); 2 cultivos de material purulento tomados por punción por piel sana, ambos positivos (1 Streptococcus pyogenes y 1 SAMS); 1 hisopado de fauces con aislamiento de Streptococcus pyogenes. Todos los que tuvieron bacteriemia eran inmunocompetentes, presentaban IPPB y tenían un recuento de leucocitos significativamente más elevado que el resto $19200 \pm 4487 / \mathrm{mm}^{3}$ versus $10679 \pm 747 / \mathrm{mm}^{3}$ $(p=0,004)$. Los valores de glóbulos blancos $\geq 11900 / \mathrm{mm}^{3}$ se asociaron a bacteriemia con un $100 \%$ de sensibilidad y $69 \%$ de especificidad; recuentos $\geq 12900 / \mathrm{mm}^{3}$ mostraron una sensibilidad de $80 \%$ y una especificidad de $72,1 \%$ (área bajo la curva de 0,84; IC $95 \%$ : 0,71-0,97).

La mediana de días de internación fue de 4 (RIC: 2-6), significativamente más prolongada en niños con inmunodeficiencia (mediana de 3 [RIC: 2-6] vs. 6 [RIC: 5-8]; $p=0,017$ ). Solo un paciente que presentó encefalitis, previamente sano, requirió cuidados intensivos. No hubo fallecidos.

La Tabla 3 resume las características clínicas, de laboratorio y la evolución de los pacientes internados.

\section{DISCUSIÓN}

Según los datos de la Organización Mundial de la Salud, la información sobre la carga de enfermedad de varicela, la incidencia y las consecuencias de las infecciones secundarias, en países en desarrollo, es limitada. ${ }^{10}$

Si bien la varicela es una enfermedad de notificación obligatoria en Argentina, ${ }^{11}$ existe un 
importante subregistro; un sustancial número de casos no se notifica por no concurrir o no tener acceso al sistema de salud, o bien, por ser asistidos en centros públicos o privados que no reportan en forma sistemática al sistema de vigilancia ante casos, especialmente, de manejo ambulatorio. $^{12}$

Este estudio aporta información acerca del impacto que las infecciones por varicela provocan en nuestro medio. Los datos respecto a las $\mathrm{TH}$ global (que incluyeron a los inmunocomprometidos) y en inmunocompetentes fueron obtenidos de tres centros de distintas modalidades de atención, complejidad y áreas del país, y el haber incluido un período de tres años consecutivos permitió disminuir el sesgo vinculado a posibles variaciones interanuales.

Nuestros resultados muestran que la varicela genera una considerable demanda hospitalaria, en especial, en niños previamente sanos: se asistió un promedio de 22 consultas ambulatorias por varicela/semana/centro. La mayoría de estas $(99,2 \%)$ y de las internaciones $(87,6 \%)$ se dieron en pacientes previamente sanos.

La proporción de casos de varicela hospitalizados sobre el total de casos registrados fue de $4,9 \%$ (IC $95 \%: 4,2-5,6$ ), similar a lo publicado en un reciente metaanálisis que evaluó la incidencia de esta enfermedad en Latinoamérica y Caribe, de 5,8 \% (2,6-10,1\%). ${ }^{12}$ Este mismo estudio reportó una tasa de internación en menores de 16 años de 43 casos (IC $95 \%$ : 24-66)/ 10000 hospitalizaciones / año, un tercio más baja que la estimada en nuestra investigación. En Holanda, otro país donde no está incorporada la vacuna, Van Lier y col. ${ }^{13}$ reportaron una incidencia similar a la observada en nuestro medio, de 68 casos / 10000 hospitalizaciones / año en menores de 16 años. En Chile, se informaron TH que fluctuaban entre 14,8 y 16,3/1000 casos notificados y, en Panamá, cifras 5 veces más altas que las reportadas en países desarrollados. ${ }^{2,8}$ Es probable que la subnotificación de casos y las dificultades para una vigilancia activa expliquen, en parte, esta variabilidad. $2,8,12,14$

Antes de contar con la disponibilidad de la vacuna en Estados Unidos, cada año, se presentaban alrededor de cuatro millones de casos..$^{2,3}$ De ellos, unos 10000 necesitaban hospitalización (25 cada 10000 ) y 100 fallecían. En ese país, varias publicaciones estimaron beneficiosa la vacunación infantil universal contra la varicela, con una razón beneficio/ costo de 2,1 a $6,9.8,15$

Son escasas las investigaciones disponibles que evalúan el impacto diferencial entre la infección en niños con y sin comorbilidades. Un estudio realizado en Turquía en la era prevacunación reportó una TH global en menores de 17 años de $107 / 10000$, mientras que $87 / 10000$ niños eran previamente sanos, lo que comprendió el $81,6 \%{ }^{4}$ En nuestra población, la tasa de internación en niños previamente sanos resultó un poco inferior $(12,4 \%)$ a la tasa global.

En países donde se ha incorporado la vacuna, se ha evidenciado una gran disminución en

TABla 3. Características clínicas, de laboratorio y evolución de los pacientes internados $(n=163)$

\begin{tabular}{|c|c|c|c|}
\hline Variable & Inmunocompetentes $(n=134)$ & Inmunocomprometidos $(n=19)$ & $p$ \\
\hline Edad en meses, $\mathrm{m} \pm \mathrm{DE}$ & $40 \pm 38$ & $78 \pm 54$ & $<0,001$ \\
\hline Sexo masculino, n (\%) & $69(51,4)$ & $11(57,9)$ & ns \\
\hline Contacto conocido, n (\%) & $42(31,3)$ & $8(42,1)$ & ns \\
\hline $2^{\circ}$ contacto intrafamiliar, $\mathrm{n}(\%)$ & $31(23,1)$ & $7(36,8)$ & ns \\
\hline \multicolumn{4}{|l|}{ Parámetros de laboratorio } \\
\hline Leucocitos $\left(/ \mathrm{mm}^{3}\right), \mathrm{m} \pm \mathrm{DE}$ & $11059 \pm 5831(n=121)$ & $7066 \pm 3637(n=17)$ & 0,007 \\
\hline $\mathrm{AST}>80 \mathrm{U} / \mathrm{L}, \mathrm{n}(\%)$ & $5 / 45(11,1)$ & $4 / 14(28,6)$ & ns \\
\hline ALT > $80 \mathrm{U} / \mathrm{L}, \mathrm{n}(\%)$ & $6 / 46(13)$ & $3 / 14(21,4)$ & ns \\
\hline \multicolumn{4}{|l|}{ Evolución } \\
\hline Días de evolución previa, $\mathrm{m} \pm \mathrm{DE}$ & $4,3 \pm 2,5$ & $2,1 \pm 1,3$ & $<0,001$ \\
\hline Con complicación, n (\%) & $134(100)$ & $7(36,8)$ & - \\
\hline Días totales de fiebre, $\mathrm{m} \pm \mathrm{DE}$ & $2,4 \pm 1,9(\mathrm{n}=107)$ & $2,2 \pm 2,0(n=13)$ & ns \\
\hline Días totales de internación, $\mathrm{m} \pm \mathrm{DE}$ & $4,3 \pm 3,3$ & $6,2 \pm 2,5$ & 0,017 \\
\hline Días totales de antibióticos, $\mathrm{m} \pm \mathrm{DE}$ & $9,7 \pm 2,8(\mathrm{n}=94)$ & $10,4 \pm 3,9(\mathrm{n}=9)$ & ns \\
\hline Días totales de aciclovir, $\mathrm{m} \pm \mathrm{DE}$ & $6,9 \pm 3,6(n=51)$ & $7,3 \pm 1,9(\mathrm{n}=18)$ & ns \\
\hline
\end{tabular}

m: media; DE: desvío estándar; ns: no significativo; AST: aspartato aminotransferasa, ALT: alanina aminotransferasa. 
la incidencia de casos de varicela como en su morbimortalidad. ${ }^{16-18}$ En España, aquellas comunidades que decidieron vacunar a los adolescentes e infantes evidenciaron que la $\mathrm{TH}$ media pasó de 39 a 21 ingresos / 100000 habitantes, con una reducción del $43 \%$ en el período posvacunal. ${ }^{19}$

En nuestra región, el primer país latinoamericano en introducir la vacuna a su calendario oficial fue la República del Uruguay, donde se reportó una disminución del $87 \%$ de las consultas ambulatorias y un $81 \%$ de las internaciones. ${ }^{18,20}$

En nuestra investigación, se observó que más de la mitad de las consultas ambulatorias $(57,6 \%)$ e internaciones $(65 \%)$ ocurrieron en menores de 5 años, dato similar al que se presentó en Estados Unidos antes de la introducción de la vacuna. ${ }^{21,22}$ En nuestra población, se observó que los menores de un año y los mayores de 14 tuvieron 1,7 y 3,7 veces más riesgo de internarse que el resto de los casos.

Si bien la varicela no está contemplada como problemática de prioridad en salud pública, ${ }^{10}$ no debe subestimarse el impacto socioeconómico que genera una enfermedad que afecta prácticamente a todos los niños y obliga a su ausentismo escolar y al ausentismo laboral de las personas encargadas de cuidarlos. El tiempo de estadía hospitalaria de nuestros pacientes fue menor que lo reportado en Panamá, ${ }^{9}$ aunque similar a lo descrito en otros países. ${ }^{4,12,21}$ La mediana de internación en nuestro medio fue de 4 días (RIC: de 2 a 9 días). Valentim y col. estimaron, en Brasil, entre 5 y 15 días de trabajo perdidos en los padres de niños internados. ${ }^{23}$

Un hallazgo llamativo fue que, aproximadamente, un tercio de los pacientes tuvieron una fuente de contagio conocida y solo el $6 \%$ recibió algún tipo de profilaxis secundaria, a pesar de existir recomendaciones nacionales e internacionales al respecto. ${ }^{1,9,24,25}$ Las IPPB, las afecciones respiratorias y los trastornos neurológicos fueron las principales complicaciones en nuestros pacientes. Cabe destacar el predominio de las IPPB (el $80 \%$ en ambulatorios y el $61 \%$ en internados). La frecuencia descrita de esta complicación en la bibliografía varía de $3 \%$ a $61 \%$, menor que la observada en nuestro estudio. ${ }^{2-4,7,8,12,15}$

La prevalencia de bacteriemia fue de $7,5 \%$; todos los niños que la padecieron eran inmunocompetentes y presentaban IPPB. Un recuento de leucocitos mayor de $11900 / \mathrm{mm}^{3}$ al ingresar permitiría predecirla con una alta sensibilidad $(100 \%)$ y aceptable especificidad $(69 \%)$. Los principales microorganismos aislados fueron SAMR y Streptococcus pyogenes. La sobreinfección bacteriana por Streptococcus pyogenes en niños con varicela se ha reportado en diversas partes del mundo. ${ }^{2,6} \mathrm{La}$ emergencia de SAMR y su eventual relación con mayor gravedad debe ser considerada al definir la antibioticoterapia empírica inicial. ${ }^{26}$

Los niños inmunocomprometidos que consultaron con varicela fueron internados; menos de la mitad presentó complicaciones; el 94,7\% recibió aciclovir endovenoso con una mediana de 7 días de tratamiento. Algunos autores proponen el tratamiento oral y ambulatorio en pacientes con factores de riesgo, sin complicaciones y con adecuadas posibilidades de seguimiento. ${ }^{27-29}$

Este estudio representa una importante línea de base para evaluar posteriormente el impacto de la estrategia nacional de vacunación, en especial, sobre la demanda al sistema de salud, así como sobre la morbimortalidad de esta patología en nuestro medio.

\section{CONCLUSIONES}

La carga de enfermedad registrada en nuestro estudio fue significativa en los 3 centros asistenciales evaluados, lo que generó una relevante demanda hospitalaria con $\mathrm{TH}$ considerables.

El mayor impacto se evidenció en pacientes sin comorbilidades. La mayoría de los casos se presentó en menores de 5 años. Las complicaciones por varicela ocurrieron, sobre todo, en menores de 1 año y adolescentes previamente sanos.

\section{REFERENCIAS}

1. American Academy of Pediatrics. Varicella-Zoster Infections. In: Pickering LK, Baker CJ, Kimberlin DW, Long SS, eds. Red Book. 2012. Report of the Committee on Infectious Diseases. $2^{\text {th }}$ ed. Elk Grove Village, IL: American Academy of Pediatrics; 2012.Págs.774-89.

2. Abarca K, Hirsch T, Potin M, et al. Complicaciones en niños con varicela en cuatro hospitales de Santiago - Chile: espectro clínico y estimación de costos. Rev Med Chile. 2001; 129(4):397-404.

3. Choo PW, Donahue JG, Manson JA, Platt R. The epidemiology of varicella and its complications. J Infect Dis. 1995; 172(3):706-12.

4. Özdemir H, Çandır MO, Karbuz A, et al. Chickenpox complications, incidence and financial burden in previously healthy children and those with an underlying disease in Ankara in the pre-vaccination period. Turk J Pediatr. 2011; 53(6):614-25.

5. Pierik JG, Gumbs PD, Fortanier SA, et al. Epidemiological characteristics and societal burden of varicella zoster virus 
in the Netherlands. BMC Infect Dis. 2012; 12:110.

6. Aebi C, Ahmed A, Ramilo O. Bacterial complications of primary varicella in children. Clin Infect Dis. 1996; 23(4):698-705.

7. Jackson MA, Burry VF, Olson LLC. Complications of varicella requiring hospitalization in previously healthy children. Pediatr Infect Dis J. 1992; 11(6):441-5.

8. Sáez-Llorens X, de Suman O, de Morós D, Rubio MP. Complicaciones y costos asociados a varicela en niños inmunocompetentes. Rev Panam Salud Pública. 2002; 12(2):111-6.

9. Dirección deControl deEnfermedades Inmunoprevenibles, Ministerio de Salud de la Nación, Argentina. Lineamientos técnicos: Fundamentos de la Introducción de la Vacuna contra Varicela al Calendario Nacional de Inmunizaciones 2015. [Consulta: 15 de enero de 2018]. Disponible en: http:/ / www.msal.gob.ar/images/stories/bes/ graficos / 0000000774cnt-2015-04_lineamientos-varicela.pdf.

10. Organización Mundial delaSalud. Vacunas contra varicela. [Consulta: 15 de diciembre de2017]. Disponible en:http:/ / www.who.int/immunization/Varicella_spanish.pdf.

11. Ministerio de Justicia y Derechos Humanos. Ley 15.465. Notificación Obligatoria de los casos de enfermedades infecciosas. Buenos Aires, Argentina: 24 de octubre de 1960. [Consulta: 15 de enero de 2018]. Disponible en: http:/ / servicios.infoleg.gob.ar/infolegInternet/ anexos /195000-199999/195093/norma.htm.

12. Bardach A, Cafferata ML, Klein K, et al. Incidence and Use of Resources for Chickenpox and Herpes Zoster in Latin America and the Caribbean - A Systematic Review and Meta-analysis. Pediatr Infect Dis J. 2012; 31(12):1263-8.

13. Van Lier A, van der Maas NA, Rodenburg GD, et al. Hospitalization due to varicella in the Netherlands. BMC Infect Dis. 2011; 11:85.

14. Ministerio de Salud de Chile, Departamento de Epidemiología. Varicela. Situación epidemiológica, Chile 2007-2013. [Consulta: 20 de diciembre de 2017]. Disponible en: http: / / www.minsal.cl/epidemiologia.

15. Lieu TA, Cochi SL, Black SB, et al. Cost effectiveness of a routine varicella vaccination program for US children. JAMA. 1994; 271(15):375-81.

16. Heininger U, Seward JF. Varicella. Lancet. 2006; 368(9544):1365-76.

17. Chaves SS, López AS, Watson TL, et al. Varicella in infants after implementation of the US varicella vaccination program. Pediatrics. 2011; 128(6):1071-7.

18. Quian J, Rüttimann R, Romero C, et al. Impact of universal varicella vaccination on 1-year-olds in Uruguay: 1997-2005. Arch Dis Child. 2008; 93(10):845-50.

19. Ciaravino G, Sagrado MJ, Martínez de Aragón M, et al. Informe sobre la situación de la varicela y del herpes zóster en España, 1999-2012. Madrid: Centro Nacional de Epidemiología, 2014. [Consulta: 15 de diciembre de 2017]. Disponible en: http:/ / www.isciii.es/ISCIII/es/ contenidos/fd-servicios-cientifico-tecnicos/fd-vigilanciasalertas / fd-enfermedades / fd-enfermedades-preveniblesvacunacion/InformeVaricela_HZ_1998-2012.pdf.

20. Ulloa-Gutiérrez R, Miño G, Odio C, et al. Vaccinepreventable diseases and their impact on Latin American children. Expert Rev Vaccines. 2011; 10(12):1671-3.

21. Galil K, Brown C, Lin F, Seward J. Hospitalizations for varicella in the United States, 1988 to 1999. Pediatr Infect Dis J. 2002; 21(10):931-5.

22. Davis MM, Patel MS, Gebremariam A. Decline in varicellarelated hospitalizations and expenditures for children and adults after introduction of varicella vaccine in the United States. Pediatrics. 2004; 114(3):786-92.

23. Valentim J, Sartori AM, de Soárez PC, et al. Costeffectiveness analysis of universal childhood vaccination against varicella in Brazil. Vaccine. 2008; 26(49):6281-91.

24. Paganini H. Varicela. En: Paganini H. Infectología Pediátrica. Buenos Aires: Interamericana; 2007.Págs.1098-105.

25. Marin M, Güris D, Chaves SS, et al. Prevention of Varicella: Recomendations of the Advisory Committee on Immunization Practices (ACIP). MMWR Recomm Rep. 2007; 56(RR-4):1-40.

26. Raulin O, Durand G, Gillet Y, et al. Toxin profiling of Staphylococcus aureus strains involved in varicella superinfection. J Clin Microbiol. 2010; 48(5):1696-700.

27. Rodríguez BrieschkeM, Sarkis C, Rodríguez T, etal. Varicela en el niño inmunocomprometido en la era del aciclovir. Arch Argent Pediatr. 2004; 102(1):8-12.

28. Marcó del Pont J. Varicela: nuevas conductas frente a una enfermedad común en pediatría. Arch Argent Pediatr. 2004;102(1):5-7.

29. Luedicke N, Pueyo S, Rezzonico G, Lancieri I. Evolución de la infección por virus varicela-zóster en niños infectados perinatalmente con el virus de la inmunodeficiencia humana. Arch Argent Pediatr. 2004; 102(1):18-21. 